\title{
AN ASYMPTOTIC FUNDAMENTAL SOLUTION OF THE REDUCED WAVE EQUATION ON A SURFACE*
}

\author{
BY \\ C. R. STEELE \\ Stanford University
}

\begin{abstract}
The physical problem of steady-state heat conduction in a thin shell is described by the "reduced wave equation" in which the differential operator is the (generally noneuclidean) Laplacian for the surface. A similar equation gives the approximation for steady-state waves in a prestressed curved membrane. A modification of the "geometric optics" asymptotic expansion, involving a Bessel function, is given for the fundamental point source solution. This is proven to be uniformly valid in the large, until a "caustic" is reached. Various features of the solution for a surface, which do not occur for the plane, are discussed.

1. Introduction. The intention of this investigation is to increase the understanding of the behavior of thin shells. In particular, we are concerned with the effects of geometry in the large of some arbitrary, but smooth, surface. Since the equations from a complete theory for the statics and dynamics of thin elastic shells are rather elaborate, a simpler equation which retains many of the significant features is treated. This is the reduced wave equation for a surface. The reduced wave equation for a plane is, of course, of vital importance in many physical problems, and a great deal is known about the behavior of the solutions, even for a nonhomogeneous medium, as in the text by Brekhovskikh [3].

The generalization to a surface, i.e. a two-dimensional noneuclidean space, is not, however, devoid of physical interest. The problem of heat conduction in a thin shell leads to the reduced wave equation on the surface, but with a change in sign due to the dissipation of heat into the environment along the surface. The equation was derived by Bolotin [2] and has been used for the investigation of the elastic stresses in a shallow sphere due to a concentrated impulse of heat by Nordgren and Naghdi [8] and for a general distribution by Nordgren [9]. An alternate derivation using a Legendre polynomial expansion is found in the thesis by Yang [12], who determined the elastic stresses due to impulsive line heating of a general surface using asymptotic methods. It should be mentioned, however, that practical problems are infrequently encountered in which the heating is so localized that the conduction along the surface is important. Generally the shell just assumes the local environment temperature, or it is the conduction through the shell wall thickness that is important.

As the obvious extension of the equation for a vibrating plane membrane, the reduced wave equation on a surface provides an approximation for transverse waves in a highly prestressed curved shell surface. Generally, however, the magnitude of the
\end{abstract}

\footnotetext{
* Received August 28, 1970; revised version received January 15, 1971. This work was performed with the support of National Science Foundation Grant GK2701 to Stanford University.
} 
prestress will depend on the direction at each point. So not only is the surface geometry encountered, but also an anisotropic wave speed.

Another instance in which the reduced wave equation, or rather "geometric optics", on a surface arises is in the diffraction of three-dimensional waves due to a solid body, as discussed by Lewis, Bleistein, and Ludwig [7]. These authors utilize a result obtained independently by Ludwig and Kravtsov [5] for an asymptotic expansion which is uniformly valid in a region of $E_{2}$ or $E_{3}$ containing a caustic, a line on which the exponential "geometric optics" expansion is singular. The geometric optics solution is also not valid in the neighborhood of a source point. Avila and Keller [1] used the method of matched asymptotic expansions to connect the Bessel function solution at a source point in $E_{2}$, similar to the solution used in [8], [9], to the geometric optics solution valid away from the source.

In the present paper, a Bessel function solution is obtained which is uniformly valid for a surface containing a source point. The difficulty in using the solution is, as for the plane, in the determination of the rays, which are the characteristics of the surface eiconel equation. For constant wave speed, the rays are the geodesics on the surface. Once the rays emitting from the source point are known, however, all remaining parts of the solution can be obtained by simple integrations.

In Sec. 2 an error estimate for the one-term solution for the heat conduction equation (isotropic, but inhomogeneous) is obtained. This solution is valid everywhere on a surface on which a function, involving the Gaussian curvature of the surface and the gradient of the "wave speed", is nonpositive. When this function is positive, then an envelope of the rays (a caustic) can occur at some distance from the source. On a caustic the present solution becomes singular; however, the correction can be made with the Airy function solution of [5], [7].

In Sec. 3 a formal asymptotic expansion is obtained for the prestressed membrane waves (anisotropic, inhomogeneous). All terms in the expansion are shown to be wellbehaved in the region containing the source point.

For the notation used, the analysis of Sec. 2 can be done entirely in orthogonal coordinates, so the Lamé parameters are used, which make some things clearer. For Sec. 3, however, the natural coordinates turn out to be nonorthogonal so tensors are utilized, which clarify other aspects of the problem.

2. Heat conduction equation. The equation we first consider is

$$
L[u] \equiv \Delta u-\left(\lambda^{2} C^{-2}+D\right) u=-\lambda^{2} C^{-2} f
$$

where $\lambda$ is a constant, $C, D$, and $f$ are prescribed functions, and $\Delta$ is the Laplacian operator for a region $\Sigma$ of a smooth surface imbedded in $E_{3}$. For the present investigation it is sufficient to consider only orthogonal coordinate lines on $\Sigma$, with parameters $\alpha_{1}$ and $\alpha_{2}$, for which the first fundamental form is

$$
d s^{2}=A_{1}^{2} d \alpha_{1}^{2}+A_{2}^{2} d \alpha_{2}^{2},
$$

in which $A_{1}$ and $A_{2}$ are the Lamé parameters [11]. The differential operators are then

$$
\begin{gathered}
\Delta u=\frac{1}{A_{1} A_{2}}\left[\frac{\partial}{\partial \alpha_{1}}\left(\frac{A_{2}}{A_{1}} \frac{\partial u}{\partial \alpha_{1}}\right)+\frac{\partial}{\partial \alpha_{2}}\left(\frac{A_{1}}{A_{2}} \frac{\partial u}{\partial \alpha_{2}}\right)\right], \\
\nabla B \cdot \nabla u=\frac{1}{A_{1}^{2}} \frac{\partial B}{\partial \alpha_{1}} \frac{\partial u}{\partial \alpha_{1}}+\frac{1}{A_{2}^{2}} \frac{\partial B}{\partial \alpha_{2}} \frac{\partial u}{\partial \alpha_{2}} .
\end{gathered}
$$


The general problem is to find the solution of Eq. (2.1) with prescribed conditions on $u$ or its normal derivative on the boundary curve $\Gamma$ of $\Sigma$. Because of the obvious difficulties with "exact" solutions, a result asymptotically valid as $\lambda$ becomes large is sought. For the heat conduction problem $\lambda$ is inversely proportional to a power of the shell thickness and therefore tends to be large for thin shells, for which the approximations leading to (2.1) are valid.

Numerous investigations of asymptotic behavior of solutions of partial differential equations have utilized an expansion due to Debye [Ann. Phys. 35, 1911] in which an exponential function with a large argument is of dominant importance. However, a satisfactory error estimate has apparently not been obtained, the leading term of the expansion becomes singular on certain lines (caustics), and generally the particular solution for only smooth functions $f$ can be readily obtained. If the source function $f$ is nonzero only in a small region, the exponential expansion can be used to obtain the solution at sufficiently far distances [1].

The problem of computing the fundamental solution for a point source is reminiscent of the problem of the ordinary differential equation with a transition point resolved by Langer [6]. At a transition point the ordinary differential equation loses all resemblance to an equation with constant coefficients. The uniformly valid asymptotic solution is formed with the solutions of the "comparison" equation which approximates the given equation in the vicinity of the transition point. For the class of equations considered in [6] the comparison equation is Bessel's equation. Similarly, near the source point the "geometric optics" exponential approximation to the solution of (2.1) loses validity. The correct solution must have the same behavior as (2.1) in the neighborhood of the source. Hence one is led to the Bessel function $K_{0}\left(\left(x^{2}+y^{2}\right)^{1 / 2}\right)$ which is the fundamental solution (in fact the Green's function for the infinite domain) for the equation $-v_{x x}$ $v_{y y}+v=0$, which approximates (2.1) in the neighborhood of a given point with the proper choice of $x$ and $y$.

Just as the solution for the transition point problem may be obtained, the coefficient and argument of the comparison equation solution are replaced, for the moment, by arbitrary functions $\psi$ and $\zeta$. So we consider the function

$$
v\left(\alpha_{1}, \alpha_{2}\right)=\psi\left(\alpha_{1}, \alpha_{2}\right) K_{0}\left[\lambda \zeta\left(\alpha_{1}, \alpha_{2}\right)\right] .
$$

The operator (2.1) acting on this function gives

$$
\begin{aligned}
L[v]= & K_{0}^{\prime}(\lambda \zeta)[2 \lambda \nabla \psi \cdot \nabla \zeta+\lambda \psi \Delta \zeta-\psi \lambda(\nabla \zeta \cdot \nabla \zeta) / \zeta] \\
& +K_{0}(\lambda \zeta)\left[-\psi \lambda^{2}\left(C^{-2}-\nabla \zeta \cdot \nabla \zeta\right)+\Delta \psi-\psi D\right] .
\end{aligned}
$$

The functions $\zeta$ and $\psi$ are now chosen to reduce the magnitude of (2.4).

Eiconal equation. The function $\zeta$ is chosen to make zero the terms multiplied by the highest power of $\lambda$. This gives the first-order equation

$$
\nabla \zeta \cdot \Delta \zeta=C^{-2}
$$

which is the familiar equation of geometric optics for light in a medium in which the velocity of light is $C$. The characteristics [4] satisfy the equation

$$
\alpha_{1}^{\prime \prime}+\frac{\partial A_{1}}{A_{1} \partial \alpha_{1}}\left(\alpha_{1}^{\prime}\right)^{2}+\frac{2 \partial A_{1}}{A_{1} \partial \alpha_{2}} \alpha_{1}^{\prime} \alpha_{2}^{\prime}-\frac{A_{2} \partial A_{2}}{A_{1}^{2} \partial \alpha_{1}}\left(\alpha_{2}^{\prime}\right)^{2}=-\frac{\partial C}{C^{3} A_{1}^{2} \partial \alpha_{1}}
$$


and the same equation with the subscripts 1 and 2 interchanged. Primes denote differentiation with respect to a parameter $\sigma$ related to the change in $\zeta$ and arclength $s$ along the characteristic by

$$
d \sigma=C d s=C^{2} d \zeta .
$$

Since (2.5) states that $d s=C d \zeta$ along the gradient lines of the solution surface $\zeta=$ $\zeta\left(\alpha_{1}, \alpha_{2}\right)$, the "gradient lines" must be characteristics.

Because of the analogy with geometric optics the characteristics, which for the present problem turn out to be "temperature paths", will be called "rays". For $C \equiv 1$, the rays are the geodesics on $\Sigma$, which when $\Sigma$ is a plane surface are straight lines. Generally, the ray passing through a point $P$ of $\Sigma$ at a given angle can be determined uniquely. When using the exponential expansion, it is often useful to consider the rays which intersect the boundary curve at a prescribed angle as indicated in Fig. 1a. For the present solution (2.3), the rays emitting from a source point are considered, as shown in Figs. 1b and 1c. It is convenient to form the polar coordinate system consisting of the rays, on which $\zeta$ increases, and their orthogonal trajectories, on which $\zeta$ is constant. The effect of variable $C$ on the rays for the plane is extensively discussed by Brekhovskikh [3]. A similar effect occurs for a surface; the rays diverge more rapidly than the geodesics in the direction of increasing $C$.

For completeness, we note that the rays connecting points $P$ and $Q$ of $\Sigma$ also satisfy the variational problem of finding the paths which provide stationary values of the integral

$$
I=\int_{P}^{Q} \frac{d s}{C}
$$

On such a path $\zeta_{P O}=I$.

Polar ray coordinates. A natural choice for coordinates is $\zeta$ and $\beta$ which is the angle, measured from a reference line, at which a ray passes through $P$. The metric at a point $Q$ in terms of the coordinates with origin at $P$ is

$$
d s_{\Theta}^{2}=C_{Q}^{2}\left(d \zeta_{P Q}^{2}+G_{P Q}^{2} d \beta_{P Q}^{2}\right) .
$$

When $C \equiv 1$ this is the metric of the geodesic polar coordinate system, and the lines of constant $\zeta$ are the "geodesic parallel circles" [11]. When only the single coordinate system with origin $P$ is considered, the $P$ and $Q$ subscripts will be omitted.

The function $G=G(\zeta, \beta)$ can be computed from the equation for the Gaussian curvature of the surface

$$
-\varkappa=\frac{1}{C^{2} G}\left[\frac{\partial}{\partial \zeta}\left(\frac{\partial G C}{C \partial \zeta}\right)+\frac{\partial}{\partial \beta}\left(\frac{\partial C}{C G \partial \beta}\right)\right] .
$$

which is, however, a nonlinear partial differential equation. The Laplacian of $C$, in the coordinates (2.6), is

$$
\Delta C=\frac{1}{C^{2} G}\left[\frac{\partial}{\partial \zeta}\left(G \frac{\partial C}{\partial \zeta}\right)+\frac{\partial}{\partial \beta}\left(\frac{\partial C}{G \partial \beta}\right)\right] .
$$

So the explicit dependence on $\beta$ may be eliminated by taking the sum

$$
G C(C K+\Delta C)=-\frac{\partial^{2} G}{\partial \zeta^{2}}+(i \nabla C \cdot \nabla C .
$$




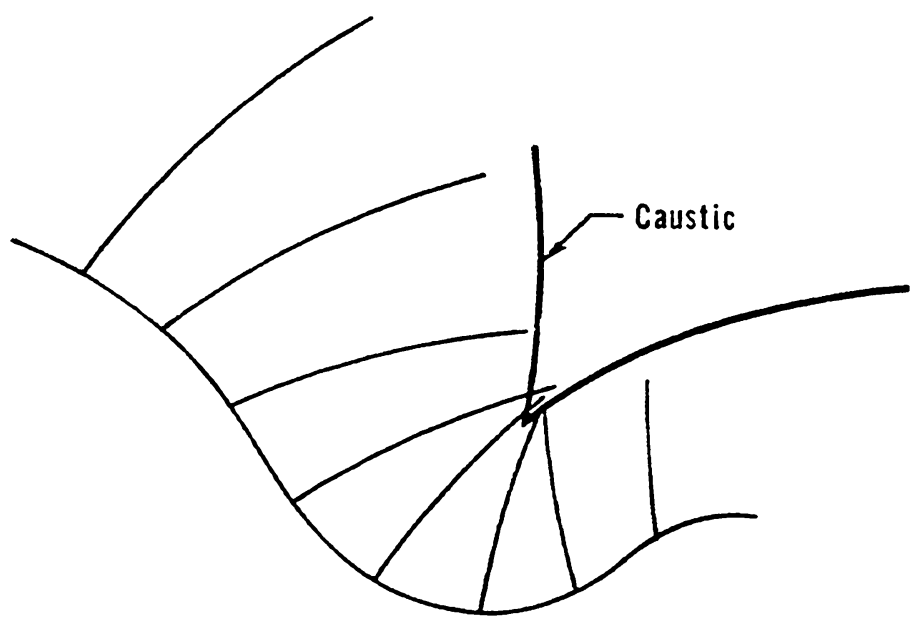

a. Rays from a Boundary Curve

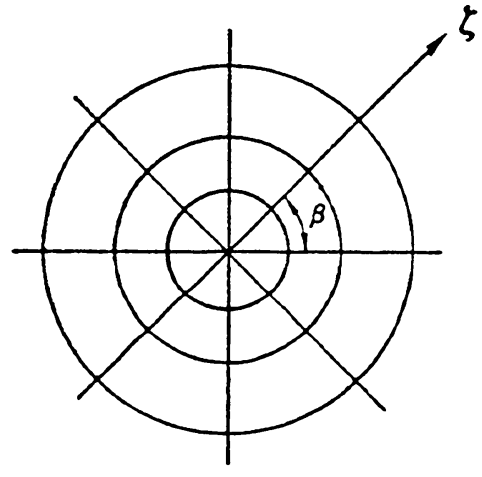

b. Rays for $C \equiv 1$ (Geodesics) for Plane

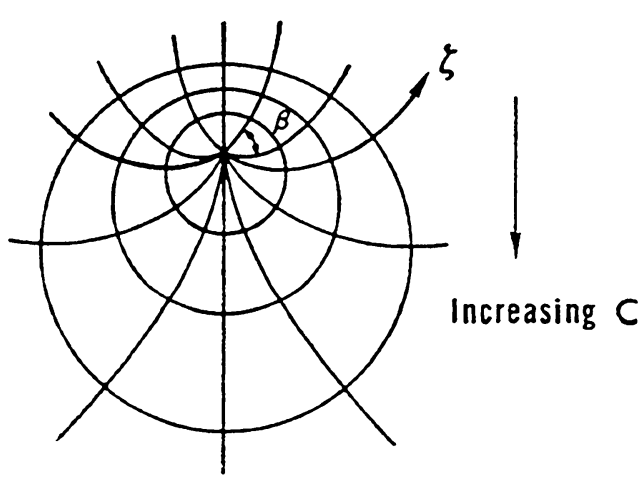

C. Rays for Variable C for Plane

Fig. 1. Rays on a surface.

Thus the ordinary linear differential equation for the variation of $G$ along a ray is obtained:

$$
\frac{\partial^{2} G}{\partial \zeta^{2}}+\mathfrak{K}^{*} G=0
$$

where the coefficient $\varkappa^{*}=C^{2} \varkappa+C \Delta C-\nabla C \cdot \nabla C$ has a value at each point of $\Sigma$ which is independent of the choice of coordinate systems. Since (2.11) is the equation 
discussed in [11] for the "geodesic radius" of the geodesic parallels of a surface $\Sigma^{*}$ with Gaussian curvature $\mathfrak{K}^{*}$ and the metric

$$
\left(d s^{*}\right)^{2}=d \zeta^{2}+G^{2} d \beta^{2}
$$

it follows that there is a conformal mapping of $\Sigma$ onto $\Sigma^{*}$ such that the rays on $\Sigma$ become geodesics on $\Sigma^{*}$ and the polar coordinates $(\zeta, \theta)$ become polar geodesic coordinates on $\Sigma^{*}$.

The behavior of geodesic polar coordinates is well known [11]. The function $G$ has the power series expansion along a given ray

$$
G_{P Q}=\zeta-\mathcal{K}_{P}^{*} \frac{\zeta^{3}}{6}+\left(\frac{\partial \mathcal{K}^{*}}{\partial \zeta}\right)_{P} \frac{\zeta^{4}}{12}+O\left(\zeta^{5}\right)
$$

so the direction of the ray affects the fourth-power term. Since $G d \theta$ is the "distance" between two "adjacent" rays, the adjacent rays intersect at the points at which $G=0$, the locus of which is an envelope of the rays from $P$, i.e. a caustic. No caustic forms if $\mathfrak{K}^{*}$ is nonpositive in $\Sigma$, since $G$ would be an increasing function of $\zeta$. Whether or not a caustic forms, the rays from $P$ will generally intersect away from $P$, and may even return to pass through $P$.

However, there is a neighborhood of $P$ in which the coordinate representation (2.9) is unique. Furthermore, Whitehead's theorem [11] states that there is a neighborhood $W^{*}$ of any point $R^{*}$ of a smooth surface $\Sigma^{*}$ that is convex, i.e. any two points of $W^{*}$ may be joined by a geodesic lying in $W^{*}$, and simple, i.e. the geodesic is unique. It follows that there exists a neighborhood $W$ of any point $R$ of the surface $\Sigma$ for which the polar coordinates (2.9) provide a unique representation of $W$ if the origin $P$ is any point in $W$. If the entire surface $\Sigma$, with the boundary curve $\Gamma$, is a $W$-neighborhood, then the behavior of the solution of (2.1) will be seen to be similar to that for a plane region with $C$ and $D$ constant. If $\Sigma$ is not a $W$-neighborhood, then complications arise because of the multiplicity of ray paths between points.

Since coordinates with origins at different points $P$ and $Q$ will be needed, we note that $G_{P Q} \equiv G_{Q P}$. This follows from the initial conditions

$$
G_{P P}=G_{\odot \odot}=0,\left.\quad \frac{\partial G_{P R}}{\partial \zeta_{P R}}\right|_{R-P}=\left.\frac{\partial G_{Q R}}{\partial \zeta_{Q R}}\right|_{R=\odot}=1,
$$

and the (self-adjoint) equation (2.11) which $G$ satisfies. Let $R$ be a point on the ray between $P$ and $Q$. Then $d \zeta_{P R}=-d \zeta_{0 R}$, so that

$$
0=\dot{x}_{Q R}\left(\frac{\partial^{2} r_{P R}}{\partial \zeta_{P R}^{2}}+K_{R}^{*} G_{P R}\right)-i_{P R}\left(\frac{\partial^{2} G_{Q R}}{\partial \zeta_{Q R}^{2}}+K_{R}^{*} G_{Q R}\right)
$$

gives

$$
0=\frac{\partial}{\partial \zeta_{P R}}\left[G_{Q R} \frac{\partial G_{P R}}{\partial \zeta_{P R}}-G_{P R} \frac{\partial G_{Q R}}{\partial \zeta_{P R}}\right] .
$$

The bracketed term must be constant; the difference in its value at $R=P$ and $R=Q$ gives the result that $G_{P Q}=G_{Q P}$.

Transport equation. The function $\psi$ of (2.3) is chosen to make zero the terms of (2.4) multiplied by $\lambda$. This gives a first-order partial differential equation but, if the coordinate system (2.9) is used, an ordinary differential equation is obtained for the variation of $\psi$ along a given ray 


$$
2 \frac{\partial \psi}{\partial \zeta}+\psi\left[\frac{1}{G} \frac{\partial G}{\partial \zeta}-\frac{1}{\zeta}\right]=0
$$

which has the solution

$$
\psi_{P Q}=\left(\zeta_{P Q} / G_{P Q}\right)^{1 / 2}=\psi_{O P} .
$$

This solution is well behaved in the neighborhood of the origin, i.e. as $Q \rightarrow P$, and only becomes singular at a caustic, at which $G=0$ but $\zeta>0$. In particular, the quantity $\Delta \psi$ is bounded, except at the caustic, and, near the coordinate origin $P$, has the expansion

$$
\Delta \psi_{P Q}=\left(\frac{\Re}{3 C^{2}}\right)_{P}-\left(\frac{\partial \mathscr{K}}{3 C^{2} \partial \zeta}\right)_{P} \zeta_{P Q}+0\left(\zeta_{P Q}^{2}\right) \text {. }
$$

When $\varkappa^{*}$ is constant the solution of $(2.11)$ is $G=\left(\varkappa^{*}\right)^{-1 / 2} \sin \left(\varkappa^{*^{1 / 2}} \zeta\right)$, which gives $\psi=\left[\mathfrak{K}^{* 1 / 2} \zeta / \sin \left(\mathcal{K}^{* 1 / 2} \zeta\right)\right]^{1 / 2}$, which has the Laplacian

$$
\psi^{-1} \Delta \psi=\frac{\mathcal{K}^{*}}{4 C^{2}}\left[1+\frac{1}{\sin ^{2}\left(\mathcal{K}^{\left.*^{1 / 2} \zeta\right)}\right.}-\frac{1}{\mathcal{K}^{*} \zeta^{2}}\right] .
$$

For $\mathfrak{K}^{*} \leq 0, \Delta \psi / \psi$ is bounded for all $\zeta$. For $\mathfrak{K}^{*}>0$ the caustic is at $\zeta=\pi \Re^{*^{-1 / 2}}$; for $0 \leq \zeta<\pi \kappa^{*-1 / 2}$ the quantity $\Delta \psi / \psi$ is bounded.

Error estimate. The choice of $\zeta$ and $\psi$ has reduced (4) to

$$
L\left[v_{P Q}\right]=f_{P Q} v_{P Q}
$$

where

$$
f_{P Q}=-D_{Q}+\frac{\Delta \psi_{P Q}}{\psi_{P Q}}, \quad v_{P Q}=\psi_{P Q} K_{0}\left(\lambda \zeta_{P Q}\right) / 2 \pi .
$$

The difference will now be estimated between $v_{P Q}$ and a function $u_{P Q}$ (the fundamental solution) which has the same logarithmic singularity at $P$ and satisfies the equation $L\left[u_{P Q}\right]=0$.

We consider first a region of a surface $\Sigma \subset W$, so that $\zeta_{P Q}, \psi_{P O}$, and hence $v_{P O}$ exist and are unique for every pair $P, Q \varepsilon \Sigma$. The function $v_{P Q}$ satisfies the requirements of a "parametrix". Thus, following the discussion in [4], $u_{P Q}$ may be written in the form

$$
u_{P Q}=v_{P Q}+\int_{\Sigma} \rho_{P R} v_{R Q} d H_{R},
$$

where $\rho_{P R}$ is an unknown function and $d H_{R}$ is the differential surface element at the point $R$,

$$
\begin{aligned}
d H_{R} & =\left(A_{1} A_{2} d \alpha_{1} d \alpha_{2}\right)_{R} \\
& =C_{R}^{2} G_{P R} d \zeta_{P R} d \beta_{P R} .
\end{aligned}
$$

The operator $L$ reduces this equation to an integral equation for $\rho_{P O}$ :

$$
\rho_{P Q}=f_{P Q} v_{P Q}+\int_{\Sigma} \rho_{P R} f_{R Q} v_{R Q} d H_{R} .
$$

If a function $z_{P Q}$ is introduced, $\rho_{P Q}=z_{P Q} v_{P Q}$, then the equation for $\rho$ becomes an equation for $z$ :

$$
z_{P Q}=f_{P Q}+\int_{\Sigma} f_{R Q} z_{P R} \frac{v_{P R} v_{R Q}}{v_{P Q}} d H_{R}
$$


If $\epsilon$ is a bound on the quantity, for all pairs $P, Q \in \Sigma$,

$$
\epsilon \geq\left|f_{P O}\right|_{\max } \int_{\Sigma}\left|\frac{v_{P R} v_{R O}}{v_{P Q}}\right| d H_{R}
$$

then we obtain $\left|z_{P O}\right| \leq\left|f_{P O}\right|+\left|f_{P O}\right|_{\max } \epsilon\left|z_{P O}\right|_{\max }$. So, if $\epsilon<1,|z|_{\max } \leq|f|_{\max } /(1-\epsilon)$.

The relation between the fundamental solution and the parametrix can be written

$$
\frac{u_{P Q}}{v_{P Q}}=1+\int_{\Sigma} z_{P R} \frac{v_{P R} u_{R O}}{v_{P Q}} d H_{R}
$$

from which the estimate is obtained

$$
\left|\frac{u_{P Q}}{v_{P O}}\right|-1 \leq|z|_{\max } \frac{\epsilon}{|f|_{\max }} \leq \frac{\epsilon}{1-\epsilon} .
$$

Therefore, if $\epsilon$ is small, the fundamental solution $u_{P Q}$ is approximated by $v_{P Q}$ uniformly for all $P, Q$ in $\Sigma$.

To show that $\epsilon$ is small, in fact is $O\left(\lambda^{-1}\right)$ as $\lambda \rightarrow \infty$, it appears to be necessary to go through the usual saddlepoint analysis of the integrals with integrands of exponential behavior. The significant behavior of the integral $I=\int_{\Sigma}\left|v_{P R} v_{R Q} / v_{P Q}\right| d H_{R}$ is retained, however, if the limiting case of the homogeneous plane is considered. Then this integral becomes (for $\lambda$ real)

$$
I=\frac{1}{2 \pi} \int_{0}^{\infty} \int_{0}^{2 \pi} \frac{K_{0}\left(\lambda \zeta_{P R}\right) K_{0}\left(\lambda \zeta_{R O}\right)}{K_{0}\left(\lambda \zeta_{P O}\right)} \zeta_{P R} d \beta d \zeta_{P R}
$$

where $\zeta_{R Q}=\left[\zeta_{P R}^{2}+\zeta_{P Q}^{2}-2 \zeta_{P R} \zeta_{P Q} \cos \beta\right]^{1 / 2}$. From the formulas in Watson [10], the result for the first integration is

$$
\int_{0}^{2 \pi} K_{0}\left(\lambda \zeta_{R Q}\right) d \beta=2 \pi K_{0}\left(\lambda \zeta_{P Q}\right) I_{0}\left(\lambda \zeta_{P R}\right)
$$

when $\zeta_{P Q}>\zeta_{P R}$, and with the $\zeta_{P Q}$ and $\zeta_{P_{R}}$ interchanged when $\zeta_{P Q}<\zeta_{P R}$. Thus $I$ is

$$
I=\int_{0}^{\zeta P Q} K_{n}(\lambda \zeta) I_{0}(\lambda \zeta) \zeta d \zeta+\frac{I_{0}\left(\lambda \zeta_{P Q}\right)}{K_{0}\left(\lambda \zeta_{P Q}\right)} \int_{\zeta P Q}^{\infty} K_{0}^{2}(\lambda \zeta) \zeta d \zeta
$$

Since we have the estimates

$$
K_{0}(x) \leq(\pi / 2 x)^{1 / 2} e^{-x}, \quad I_{0}(x) K_{0}(x) \leq 1 / 2 x,
$$

$I$ is easily found to be bounded by $I \leq\left(\zeta_{P O} / 2 \lambda\right)+\left(\pi^{2} / 4 \lambda^{2}\right)$.

For the general surface with variable $C$, the integral on the contour $\zeta_{P R}=$ const. is performed first; the significant contribution comes from the saddlepoint at $\zeta_{Q R}=$ $\left|\zeta_{P O}-\zeta_{P R}\right|$. Then the integral for $0 \leq \zeta_{P_{R}} \leq \infty$ gives the result similar to that for the plane, that $I=O\left(\zeta_{P O} / \lambda\right)$. Thus, for an finite region $\Sigma, \epsilon$ will be small for a sufficiently large value of $\lambda$, if $\nabla f_{P O}$ is bounded in $\Sigma$. The details of the estimation are tedious and follow the usual saddlepoint procedure, and so are omitted. The result may be shown to hold for complex $\lambda$, for $|\arg \lambda| \leq \pi / 2$. When $\arg \lambda=\pi / 2$, the "saddlepoints" become points of "stationary phase", so the point of maximum $\zeta_{R \circ}$ gives the same contribution as the point of minimum $\zeta_{R O}$, but the result is the same.

Thus we have

$$
u_{P Q}=v_{P O}\left[1+O\left(\lambda^{-1}\right)\right]
$$


for all $P$ and $Q$ in $\Sigma$. Thus $v_{P Q}$ is much more than a parametrix, since it provides a uniformly valid approximation to the fundamental solution despite the exponentially decreasing behavior at large values of $\operatorname{Re} \lambda \zeta_{P O}$.

Regions of multiple ray covering. If, instead of the actual surface, the Riemannian differential manifold with the metric (2.9) is considered, then the result (2.17) will hold for any simple, convex region $\Sigma$ of the manifold. However, a simple, convex region of the manifold, if referred back to the surface, may provide a multiplicity of coverings of the surface. For an example, consider a cone with $C \equiv 1$. The difference between the cone and the plane is indistinguishable in Eq. (2.1), which depends only on the metric of surface, and in the ray coordinates (2.9), which are, for this case, simply the polar coordinates in the plane. However, the ray coordinates provide a unique representation for the plane, which corresponds to the Riemannian manifold. The ray paths on the cone may be visualized, as in [11], by mapping the cone on the plane by rolling the cone on the plane with the vertex fixed, as indicated in Fig. 2. Point $Q$ of the cone touches the plane first at $Q$, then at $Q_{1}, Q_{2}, \cdots$ as the cone is rolled in one direction and at $Q_{-1}, Q_{-2}, \cdots$ as the cone is rolled in the opposite direction. Straight line rays on the plane correspond to rays on the cone which spiral inward to some minimum distance from the vertex, then spiral outward. Any straight line between $P$ and $Q_{i}$ on the plane will correspond to a ray path on the cone between $P$ and $Q$, which is different for each different $Q_{i}$.

Now the solution (2.17) is valid for any $W$-neighborhood $\Sigma_{1}$ of the cone, such as that with the boundary curve $\Gamma_{1}$ in Fig. 2. For a region $\Sigma_{2}$ of the cone which is not simply connected, such as the complete vertex portion cut by a closed curve $\Gamma_{2}$ at a constant distance $L$ from the vertex, the solution (2.17), obtained by expanding the contour $\Gamma_{1}$ so that $\Sigma_{1}$ covers $\Sigma_{2}$, will be discontinuous on the generator, the opposite sides of which two portions of $\Gamma_{1}$ will approach. However, the solution (2.17) is valid for a $W$-neighborhood of the plane such as the circle generated by the contour $\Gamma_{2}$ in Fig. 2.

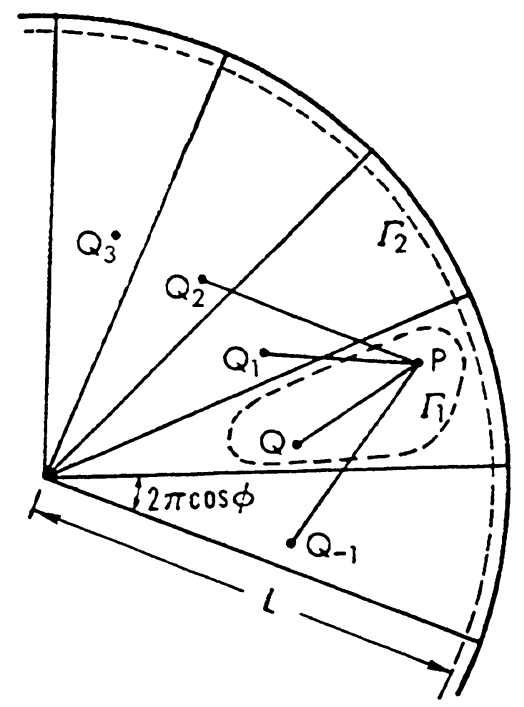

Fig. 2. Mapping of cone on the plane. 
If $\sec \varphi$, where $\varphi$ is the angle between the normal to the cone and the axis, is equal to an integer, then the multiplicity of the mappings of $\Sigma_{2}$ on the plane will exactly cover the circle of radius $L$. A solution for the plane which satisfies continuity and symmetry for each of the sectors can be obtained by placing equal sources at $P$ and its image points. Equivalently, we may take the solution for the plane with the single source at $P$, and then, for the cone, add the solutions for all of the sectors. Thus for the plane the fundamental solution is $u_{P Q}=K_{0}\left(\lambda \zeta_{P Q}\right) / 2 \pi$, but for the cone $u_{P Q}=\Sigma K_{0}\left(\lambda \zeta_{P Q_{i}}\right) / 2 \pi$, where the summation is over the distances $\zeta_{P Q_{i}}$ on all the geodesic paths connecting $P$ and $Q$.

Generally when the region $\Sigma$ of the surface is not simply connected, but free from a caustic, the fundamental solution is

$$
u_{P Q}=\frac{1}{2 \pi} \sum \psi_{P Q_{i}} K_{0}\left(\lambda \zeta_{P Q_{0}}\right)\left[1+0\left(\lambda^{-2}\right)\right]+f_{P Q}
$$

where the summation is over all the ray paths connecting $P$ and $Q$. If the number of paths is the same for all points $Q$ of $\Sigma$, such as for the cone with $\sec \varphi$ equal to an integer, the term $f_{P Q}$ may be set equal to zero. But when the number of paths is different for different regions of $\Sigma$, such as for the cone with $\sec \varphi$ not equal to an integer, then $f_{P O}$ is needed to satisfy the continuity conditions. The boundary value problem for $f_{P Q}$ may be solved by dividing $\Sigma$ into $W$-neighborhoods and using the analysis in [4].

There is also the question of convergence of the sum (2.18). A general classification of the totality of ray connections between points, which would be needed, does not seem to have been done. It does appear that for most surfaces that come to mind, the sum (2.18) is rapidly convergent and the term $f_{P Q}$ is negligible, because of the exponentially decreasing behavior of the Bessel function.

Regions not covered by rays. The opposite situation can arise where, instead of the coordinates (2.9) overlapping $\Sigma$ several times, the coordinates (2.9) may only partially cover $\Sigma$. For example, consider the surface of revolution which has the metric

$$
d s^{2}=d \rho^{2}+r^{2} d \theta^{2},
$$

where $\rho$ is the meridional arclength, $\theta$ is the circumferential angle, and $r=r(\rho)$. If $C$ is a function only of $\rho$ then the rays can be obtained by quadrature. From (2.6) the rav equations are

$$
\frac{d^{2} \rho}{d \sigma^{2}}-r r^{\prime}\left(\frac{d \theta}{d \sigma}\right)^{2}=-\frac{\partial C}{C^{3} \partial \rho}, \quad \frac{d^{2} \theta}{d \sigma^{2}}+2 \frac{r^{\prime}}{r} \frac{d \theta}{d \sigma} \frac{d \rho}{d \sigma}=-\frac{\partial C}{C^{3} r^{2} \partial \theta} .
$$

When $C=C(\rho)$, the second equation gives $d \theta / d \sigma=A r^{-2}$ where $A$ is a constant. Then the first equation is

$$
\frac{1}{2} \frac{d}{d \rho}\left(\frac{d \rho}{d \sigma}\right)^{2}=A^{2} r^{-3} r^{\prime}-C^{-3} C^{\prime}
$$

which yields $\sigma=\int_{\rho_{0}}^{\rho_{0}}\left(C^{-2}-A^{2} r^{-2}\right)^{-1 / 2} d \rho$. It is convenient to introduce $\alpha$, the angle between the rays, and the meridian $\tan \alpha=r d \theta / d \rho$, from which is obtained, with the obvious identification of the constant $A$,

$$
(r \sin \alpha) / C=A=\left(r_{0} \sin \alpha_{0}\right) / C_{0} .
$$

This reduces to the theorem of Clairaut for $C \equiv 1$, when the rays are geodesics. In terms of $\alpha=\alpha(\rho)$, we have 


$$
\begin{aligned}
& \zeta=\int C^{-2} d \sigma=\int_{\rho_{0}}^{\rho}(C \cos \alpha)^{-1} d \rho, \\
& \theta=\theta_{0}+\int_{\rho_{0}}^{\rho} \tan \alpha \cdot d \rho / r .
\end{aligned}
$$

For the ray coordinates (2.9) from the point $P$ at $\left(\rho_{0}, \theta_{0}\right)$, we have $\beta=\alpha_{0}$ and, after some manipulation,

$$
\begin{aligned}
G & =\frac{r \cos \alpha}{C} \frac{\partial \theta}{\partial \alpha_{0}}=\cot \alpha_{0} \cot \alpha \int_{\rho_{0}}^{\rho} \tan ^{2} \alpha \frac{d \rho}{C \cos \alpha} \\
& =\cot \alpha_{0} \cot \alpha \int_{0}^{\zeta} \tan ^{2} \alpha d \zeta .
\end{aligned}
$$

A particularly simple reduction of (2.19) occurs for a one-parameter family of variation in $C$. If we set

$$
\frac{d \theta}{d \alpha}=\frac{\tan \alpha}{r} \frac{d \rho}{d \alpha}=\frac{C}{r^{2}(d / d \rho)(C / r)}
$$

equal to a constant $-k$, then $C$ must have the variation

$$
C=C_{0}\left(r / r_{0}\right) \exp \left(-k \int_{\rho_{0}}^{\rho} d \rho / r\right) .
$$

For this variation the equations for the rays (2.19) become

$$
\begin{aligned}
\sin \alpha & =\sin \alpha_{0} \exp \left(-k \int_{\rho_{0}}^{\rho} d \rho / r\right), \quad \zeta=\frac{\sin \left(\alpha_{0}-\alpha\right)}{k \sin \alpha}, \\
\theta & =\theta_{0}+k\left(\alpha_{0}-\alpha\right), \quad G \equiv \zeta .
\end{aligned}
$$

Incidentally, since $\psi=(\zeta / G)^{1 / 2} \equiv 1$, the remainder term of (2.17) is identically zero when $D \equiv 0$, so the asymptotic solution turns out to be an exact solution for a nontrivial class of problems.

For a dome with $r(\rho)$ an increasing function, the rays (2.21) spiral around the dome inward to the apex, when $k$ is negative, and so give a multiplicity of ray connections between points. However, when $k$ is positive $\alpha$ decreases from the initial angle $\alpha_{0}$ to zero, which gives the rays shown in Fig. 3 . The rays from $P$ are all contained in the sector

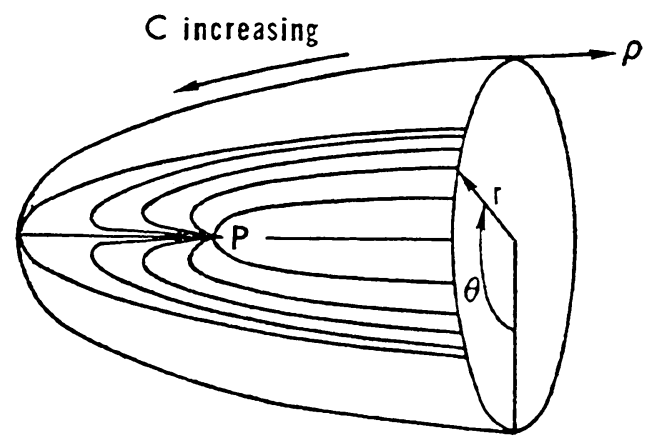

Fig. 3. Rays on a surface of revolution with increasing $C$. 
$-\pi / k \leq \theta-\theta_{0} \leq \pi / k$. Since there is a unique ray from $P$ to every point in the sector, there is no caustic. Any sector $0 \leq \theta \leq \theta_{\max }$, where $\theta_{\max } \leq \pi / k$, is a $W$-neighborhood. For a sector $\Sigma$ with $\theta_{\max }>\pi / k$, an additional function must be added to (2.17) which removes the discontinuity at $\left|\theta-\theta_{0}\right|=\pi / k$ to provide an asymptotic fundamental solution for $\Sigma$.

3. Wave equation. Under static loads, the stresses in a curved membrane satisfy the equilibrium equation $T \nabla \cdot \mathbf{N}+\mathbf{p}=0$, where $T$ is a constant giving some average membrane force per unit length, $\mathbf{N}$ is the symmetric dimensionless stress tensor, and $\mathbf{p}$ gives the prescribed vector force per unit area of the surface. If small-amplitude, transverse vibrations of this prestressed membrane are considered, then an approximate equation for the waves has the form ${ }^{1}$

$$
L[u]=\nabla \cdot(\mathbf{N} \cdot \nabla u)+\left(\mu^{2} C^{-\underline{-}}-D\right) u=0,
$$

where $\mu$ is the frequency parameter. For a membrane under an isotropic state of tension, the dimensionless stress tensor is just the identity dyad $\mathbf{N}=\boldsymbol{\delta}=\mathbf{a}^{\alpha} \otimes \mathbf{a}_{\alpha}$, in which $\mathbf{a}_{\alpha}$ and $\mathbf{a}^{\alpha}$ are the base and reciprocal base vectors, respectively, and $\otimes$ denotes the tensor product [11]. Then (3.1) reduces to the equation studied in the previous section (2.1), except that $\lambda^{2}$ has been replaced by $-\mu^{2}$, so that we now obtain oscillatory rather than exponentially-decreasing solutions. Thus the objective of this section is to determine the effect of a general $\mathbf{N}$, giving an anisotropic speed of sound. Furthermore, rather than repeat the error estimate of the one-term solution of the preceding section, we obtain the formal asymptotic expansion for the solution.

The Bessel function $J(\mu \zeta)$ is the solution for waves on the isotropic plane membrane emitting from a source point [1]. As in the preceding section, we take a function $\alpha_{0}\left(x^{\alpha}\right) J\left(\mu \zeta\left(x^{\alpha}\right)\right)$ and find that

$$
\begin{aligned}
L\left[\alpha_{0} J(\mu \zeta)\right]= & J(\mu \zeta)\left[\mu^{2}\left(C^{-2}-\nabla \zeta \cdot \mathbf{N} \cdot \nabla \zeta\right) \alpha_{0}+\nabla \cdot\left(\mathbf{N} \cdot \nabla \alpha_{0}\right)-D \alpha_{0}\right] \\
& +J^{\prime}(\mu \zeta) \mu\left[2 \nabla \alpha_{0} \cdot \mathbf{N} \cdot \nabla \zeta+\alpha_{0}(\nabla \cdot(\mathbf{N} \cdot \nabla \zeta)-(\nabla \zeta \cdot \mathbf{N} \cdot \nabla \zeta) / \zeta)\right] .
\end{aligned}
$$

Setting the term multiplied by $\mu^{2}$ to zero gives the eiconal equation

$$
\nabla \zeta \cdot \mathbf{N} \cdot \nabla \zeta=C^{-2},
$$

while the coefficient of $\mu$ gives the transport equation

$$
2 \nabla \alpha_{0} \cdot \mathbf{N} \cdot \nabla \zeta+\alpha_{0}(\nabla \cdot(\mathbf{N} \cdot \nabla \zeta)-(\nabla \zeta \cdot \mathbf{N} \cdot \nabla \zeta) / \zeta)=0 .
$$

To cancel the remaining term of (3.2) it is necessary to add another term to the function $\alpha_{0} J(\mu \zeta)$. As in the case of the ordinary differential equation, the proper term to add is the derivative of $J(\mu \zeta)$. For a function $\alpha_{i+1}$ undetermined at this point,

$$
\begin{aligned}
& L\left[\alpha_{i+1} \zeta J^{\prime}(\mu \zeta)\right] \\
& \quad=-J(\mu \zeta) \mu\left[\alpha_{i+1} \nabla \zeta \cdot \mathbf{N} \cdot \nabla \zeta+\zeta\left(\alpha_{i+1} \nabla \cdot(\mathbf{N} \cdot \nabla \zeta)+2 \nabla \alpha_{i+1} \cdot \mathbf{N} \cdot \nabla \zeta\right)\right] \\
& \quad+J^{\prime}(\mu \zeta) \zeta\left(\nabla \cdot\left(\mathbf{N} \cdot \nabla \alpha_{i+1}\right)-D \alpha_{i+1}\right) .
\end{aligned}
$$

Thus the appropriate expansion is found to be

$$
u=\sum_{i=0.2 .4, \ldots}\left[J(\mu \zeta) \alpha_{i} \mu^{-i}+\zeta J^{\prime}(\mu \zeta) \alpha_{i+1} \mu^{-i-1}\right] .
$$

1 The validity of this equation does not seem to have been established, except for the plane surface; but it has at least some of the features of the more exact equations which include the coupling with tangential motion. 
Taking $L[u]=0$ and setting the coefficient of each power of $\mu$ to zero gives the equations for the coefficients

$$
\begin{aligned}
& 2 \nabla \alpha_{i} \cdot \mathbf{N} \cdot \nabla \zeta+\alpha_{i}\left[\nabla \cdot(\mathbf{N} \cdot \nabla \zeta)-\frac{\nabla \zeta \cdot \mathbf{N} \cdot \nabla \zeta}{\zeta}\right]=\zeta\left[D \alpha_{i-1}-\nabla \cdot\left(\mathbf{N} \cdot \nabla \alpha_{i-1}\right)\right], \\
& 2 \nabla \alpha_{i+1} \cdot \mathbf{N} \cdot \nabla \zeta+\alpha_{i+1}\left[\nabla \cdot(\mathbf{N} \cdot \nabla \zeta)+\frac{\nabla \zeta \cdot \mathbf{N} \cdot \nabla \zeta}{\zeta}\right]=\frac{1}{\zeta}\left[-D \alpha_{i}+\nabla \cdot\left(\mathbf{N} \cdot \nabla \alpha_{i}\right)\right],
\end{aligned}
$$

for each value $i=0,2,4, \cdots$. Thus if $\alpha_{-1}$ is set equal to zero, with $i=0,(3.7 \mathrm{a})$ is the transport equation (3.4) and (3.7b) gives $\alpha_{1}$. Then with $i=2$, (3.7a) gives $\alpha_{2}$ and (3.7b) gives $\alpha_{3} ;$ and so on. The properties of the solution of the eiconal equation (3.3) and the system (3.7) will now be discussed.

Eiconel equation. Eq. (3.3) for the phase function is, as before, a first-order nonlinear partial differential equation

$$
F=\nabla \zeta \cdot \mathbf{N} \cdot \nabla \zeta-C^{-2}=0 .
$$

The canonical form of the equations for the characteristics is [4]

$$
\begin{aligned}
d x^{\alpha} / d \sigma & =\partial F / \partial p_{\alpha}, \\
d p_{\alpha} / d \sigma & =-\partial F / \partial x^{\alpha}, \\
d \zeta / d \sigma & =p_{\alpha}\left(\partial F / \partial p_{\alpha}\right)
\end{aligned}
$$

where $\sigma$ is the appropriate proportionality factor and where $\nabla \zeta=\mathrm{a}^{\alpha} p_{\alpha}$. A more lucid form can, however, be obtained. The tangent to the (projection of the) characteristic on the surface is obtained from (3.9a)

$$
d \mathbf{r} / d \sigma=\mathbf{a}_{\alpha}\left(d x^{\alpha} / d \sigma\right)=\partial F / \partial \nabla \zeta
$$

where $\mathrm{r}$ is the position vector to a point on the surface. The strip condition (3.9b) gives the change in the gradient along the characteristic

$$
\frac{d \nabla \zeta}{d \sigma}=-(\nabla F)_{\nabla \zeta}+\left(\nabla \zeta \cdot \mathrm{b} \cdot \frac{d \mathbf{r}}{d \sigma} \mathbf{a}_{3}\right)
$$

where $(\nabla F)_{\nabla \zeta}$ is the gradient of $F$ with $\nabla \zeta$ held constant. For the actual calculation of the characteristic on the surface, only the intrinsic part of $(3.10 \mathrm{~b})$ is needed: $\delta \cdot(d \nabla \zeta / d \sigma)$ $=-(\nabla F)_{\nabla \zeta}$. For the variation in the solution along the characteristic, (3.9c) is

$$
\frac{d \zeta}{d \sigma}=\frac{\partial F}{\partial \nabla \zeta} \cdot \nabla \zeta .
$$

Using (3.10), the characteristics of Eq. (3.8) are found to be described by

$$
\begin{aligned}
& \frac{d \mathbf{r}}{d \sigma}=2 \mathbf{N} \cdot \nabla \zeta, \quad \mathbf{\delta} \cdot \frac{d \nabla \zeta}{d \sigma}=-\left(\nabla \zeta \cdot \frac{\partial \mathbf{N}}{\partial x^{\alpha}} \cdot \nabla \zeta\right) \mathbf{a}^{a}, \\
& \frac{d \zeta}{d \sigma}=2 \nabla \zeta \cdot \mathbf{N} \cdot \nabla \zeta=\frac{2}{C^{2}} .
\end{aligned}
$$

From (3.11a) the relation between $\sigma$ and the arclength along the characteristic is obtained:

$$
(d r / d \sigma)^{2}=4 \nabla \zeta \cdot \mathbf{N} \cdot \mathbf{N} \cdot \nabla \zeta .
$$


If $\nabla \zeta$ is prescribed at a point on the surface, (3.11a) gives the direction of the characteristic, and (3.11d) gives the increment in distance with an increment in $\sigma$, then (3.11c) gives the increment in $\nabla \zeta$. Thus a direct step-by-step numerical procedure can be used for the computation of the characteristic curve on the surface, after which the value of the solution $\zeta$ requires a simple integration (3.11c).

Polar coordinates. It now is obvious that each of the set (3.7) also reduces to a first-order ordinary differential equation along a characteristic. The physical interpretation is that energy is propagated along the characteristics (rays). Thus an obvious choice for coordinate curves on the surface are the rays emitting from the source point $P$, as was done in the previous section. Now, however, the rays are not orthogonal to the contour lines, i.e. the lines of constant $\zeta$. Thus the rays and wave fronts are not orthogonal, as before, but still provide the natural coordinate system to use.

Therefore we choose

$$
x^{1}=\zeta, \quad x^{2}=\beta,
$$

where $\beta$ is some measure of the angle between the rays from the source point $P$ and some fixed line. The constant of integration in (3.11c) and the sign of $\sigma$ are chosen so that $\zeta=0$ at $P$ and increases with the distance from $P$. The base vectors are

$$
\begin{aligned}
& \mathbf{a}_{1}=\frac{\partial \mathbf{r}}{\partial \zeta}=\left(\frac{d \mathbf{r}}{d \sigma}\right) \frac{d \sigma}{d \zeta}=C^{2} \mathbf{N} \cdot \nabla \zeta, \\
& \mathbf{a}_{2}=a \nabla \zeta \cdot \mathbf{\varepsilon},
\end{aligned}
$$

where

$$
a=\left[a_{11} a_{22}-a_{12}^{2}\right]^{1 / 2}=\left|a_{1} \times a_{2}\right|,
$$

and where the tensor

$$
\varepsilon=a^{-1}\left[a_{1} \otimes a_{2}-a_{2} \otimes a_{1}\right],
$$

when dotted with a tangent vector, gives a $90^{\circ}$ rotation of the vector. The reciprocal base vectors are

$$
\mathrm{a}^{1}=\nabla \zeta, \quad \mathrm{a}^{2}=-C^{2} a^{-1} \varepsilon \cdot \mathbf{N} \cdot \nabla \zeta=a^{-1} \mathrm{a}_{1} \cdot \varepsilon .
$$

To obtain the correct choice for the second coordinate $\beta$, the local behavior of the solution at the point $P$ must be considered. The stress tensor at $P$ is

$$
\mathbf{N}=\mathbf{N}_{1} \mathbf{e}_{1} \otimes \mathbf{e}_{1}+\mathbf{N}_{2} \mathbf{e}_{2} \otimes \mathbf{e}_{2},
$$

where $T N_{1}$ is the maximum tension in the direction of the unit (tangent) vector $\mathbf{e}_{1}$, and $T N_{2}$ is the minimum tension in the orthogonal direction $\mathbf{e}_{2}$. Thus on the local tangent plane, with coordinates $x_{1}$ and $x_{2}$ in the directions $\mathbf{e}_{1}$ and $\mathbf{e}_{2}$, Eq. (3.1) is

$$
N_{1} \frac{\partial^{2} u}{\partial x_{1}^{2}}+N_{2} \frac{\partial^{2} u}{\partial x_{2}^{2}}+\mu^{2} C^{-2} u=0 .
$$

In terms of a "stretched" coordinate system

$$
\bar{x}_{1}=x_{1} N_{1}^{-1 / 2}, \quad \bar{x}_{2}=x_{2} N_{2}^{-1 / 2}
$$

the equation becomes the reduced wave equation, giving the source solution

$$
u=J\left(\frac{\mu}{C}\left[\bar{x}_{1}^{2}+\bar{x}_{2}^{2}\right]^{1 / 2}\right)
$$


and the polar angle $\beta=\tan ^{-1} \bar{x}_{2} / \bar{x}_{1}$. Returning to the local physical coordinates, we have

$$
\zeta=C^{-1}\left[N_{1}^{-1} x_{1}^{2}+N_{2}^{-1} x_{2}^{2}\right]^{1 / 2}, \quad \beta=\tan ^{-1} \frac{x_{2}}{x_{1}}\left(\frac{N_{1}}{N_{2}}\right)^{1 / 2} .
$$

The transformation from the rectangular cartesian coordinates to the polar coordinates gives

$$
a=\left(\frac{\partial(\zeta, \beta)}{\partial\left(x_{1} x_{2}\right)}\right)^{-1}=\left(N_{1} N_{2}\right)^{1 / 2} C^{2} \zeta
$$

while the base vectors of the polar coordinates are, from

$$
\mathbf{a}_{1}=\zeta^{-1}\left(x_{1} \mathbf{e}_{1}+x_{2} \mathbf{e}_{2}\right), \quad \mathbf{a}_{2}=a \zeta^{-1}\left(N_{1}^{-1} x_{1} \mathbf{e}_{2}-N_{2}^{-1} x_{2} \mathbf{e}_{1}\right) C^{-2},
$$

and the reciprocal base vectors are

$$
\mathbf{a}^{1}=\nabla \zeta=C^{-2} \zeta^{-1}\left(N_{1}^{-1} x_{1} \mathbf{e}_{1}+N_{2}^{-1} x_{2} \mathbf{e}_{2}\right), \quad \mathbf{a}^{2}=(a \zeta)^{-1}\left(x_{1} \mathbf{e}_{2}-x_{2} \mathbf{e}_{1}\right) . \quad(3.15 \mathrm{f}, \mathrm{g})
$$

Thus (3.15) gives the local behavior of the $\zeta, \beta$ coordinate system at the point $P$ and so provides the appropriate initial conditions for the computation of the effects in the large.

As shown by (3.15d), the rays do emit radially from the source point. For the geodesic curvature of a ray in the large

$$
\kappa_{\diamond}=\frac{d}{d s}\left(\frac{d \mathbf{r}}{d s}\right) \cdot \delta=2\left(\frac{d \sigma}{d s}\right)^{4} \frac{d \mathbf{r}}{d \sigma} \cdot \nabla(\mathbf{N} \cdot \nabla \zeta) \cdot \varepsilon \cdot \frac{d \mathbf{r}}{d \sigma},
$$

which is not particularly illuminating. However, for isotropic tension $\mathbf{N}=\boldsymbol{\delta}$, this form readily gives $\kappa_{\vartheta}=C^{-1} \nabla C \cdot \varepsilon \cdot d \mathbf{r} / d s$, which was obtained more awkwardly in the preceding section (2.8).

Transport equation. With the ray polar coordinate system computed, the solutions of the transport equations (3.7) are very easily obtained. The equation for $\alpha_{0}(3.4)$ is

$$
\frac{2}{C^{2}} \frac{\partial \alpha_{0}}{\partial \zeta}+\alpha_{0}\left[\nabla \cdot\left(C^{-2} \mathbf{a}_{1}\right)-C^{-2} \zeta^{-1}\right]=0 .
$$

But $\nabla \cdot \mathbf{a}_{1}=\partial a / a \partial x^{1}$, so the solution is

$$
\alpha_{0}=\left(a^{-1} C^{2} \zeta\right)^{-1 / 2}
$$

Because of the behavior at the source point (3.15c) $\alpha_{0}$ is well-behaved, and becomes singular only at a caustic, on which $a=0$, which must be a finite distance away from the source point. The nonhomogeneous form (3.7a) readily gives

$$
\alpha_{i}=\frac{1}{2} \alpha_{0} \int_{0}^{\zeta}\left(a C^{2} \zeta\right)^{1 / 2}\left[D \alpha_{i-1}-\nabla \cdot\left(\mathbf{N} \cdot \alpha_{i-1}\right)\right] d \zeta .
$$

Thus if $\alpha_{i-1}$ is well behaved, so will be $\alpha_{i}$.

The equations for $\alpha_{1}, \alpha_{3}, \cdots(3.7 \mathrm{~b})$ have only a change in sign, which makes the complementary solution singular. However, only the particular solution is needed, which is well behaved:

$$
\alpha_{i+1}=\frac{1}{2}\left(C^{-2} a \zeta\right)^{-1 / 2} \int_{0}^{\zeta}\left(C^{-2} a \zeta^{-1}\right)^{1 / 2}\left[-D \alpha_{i}+\nabla \cdot\left(\mathbf{N} \cdot \nabla \alpha_{i}\right)\right] d \zeta .
$$

Thus if the metric coefficients and the prestress are analytic, then all the $\alpha_{i}$ are analytic 
in a finite region around the source point. If the coefficients and prestress have a bounded high derivative, then the $\alpha_{i}$ will be well-behaved until a certain unbounded $\alpha_{N}$ is encountered. Presumably, as in the case of the ordinary differential equation, it would be possible to truncate the series (3.6) and then proceed through an error estimation similar to that of Sec. 2, with the result that the error is of the magnitude of the first term in the series neglected. For practical purposes, however, if the second term in the series is not reasonably small in comparison with the first, then this entire approach is useless.

The one feature of the isotropic that has not been generalized to the case of anisotropic tension is the simple equation for the direct calculation of $a=\left|a_{1} \times a_{2}\right|$ along a given ray. For the isotropic case $a=C^{2} G$, and $G$ satisfies (2.11). A similar simple equation for the anisotropic case has not yet been found.

\section{References}

[1] G. S. S. Avila and J. B. Keller, The high-frequency asymptotic field of a point source in an inhomogeneous medium, Comm. Pure Appl. Math. 16, 363-381 (1963)

[2] V. V. Bolotin, Equations for the non-stationary temperaiure fields in thin shells in the presence of sources of heat, Prikl. Math. Meh. 24, 361-363 (1960) = J. Appl. Math. Mech. 24, 515-519 (1960)

[3] L. M. Brekhovskikh, Waves in layered media, Izdat. Akad. Nauk SSSR, Moscow, 1957; English transl., Academic Press, New York, 1960

[4] P. R. Garabedian, Partial differential equations, Wiley, New York, 1964

[5] Yu. A. Kravtsov, Two new asymptotic methods in the theory of wave propagation in inhomogeneous media (review), Soviet Physics-Acoustics 14, 1-17 (1968)

[6] R. E. Langer, On the asymptotic solutions of ordinary differential equations, with reference to the Stokes' phenomenon about a singular point, Trans. Amer. Math. Soc. 37, 397-416 (1935)

[7] R. M. Lewis, N. Bleistein and D. Ludwig, Uniform asymptotic theory of creeping waves, Comm. Pure Appl. Math. 20, 295-328 (1967)

[8] R. P. Nordgren and P. M. Naghdi, Propagation of thermoelastic waves in an unlimiled shallow spherical shell under heating, Proc. Fourth U. S. Nat. Congr. Appl. Mech. (Univ. of California, Berkeley, Calif., 1962), vol. 1, Amer. Soc. Mech. Engrs., New York, 1962, pp. 311-324

[9] R. P. Nordgren, On the method of Green's function in the thermoelastic theory of shallow shells, Internat. J. Engrg. Sci. 1, 279-308 (1963)

[10] G. N. Watson, A treatise on the theory of Bessel functions, 2nd ed., Cambridge Univ. Press, New York, 1944

[11] T. J. Willmore, Differential geometry, Oxford Univ. Press, London, 1959

[127 J. -S. Yang, Thermal stresses in thin shells, $\mathrm{Ph} . \mathrm{D}$. Thesis, Stanford University, 1969; see also Stanford University Report, SUDAAR No. 371, Stanford Calif., 1969 